\title{
Cooperative Microgrid Networks for Remote and Rural Areas
}

\author{
Eman Hammad, Abdallah Farraj, Deepa Kundur \\ Department of Electrical and Computer Engineering, University of Toronto, Ontario, Canada \\ Email: \{ehammad, abdallah, dkundur\}@ece.utoronto.ca
}

\begin{abstract}
Microgrids (MGs) with renewable energy resources have shown competitive operational advantages for smart grid integration. In the wake of recent blackout events, more interest has been directed on the deployment of MGs in rural and remote areas where main grid connectivity may not be possible. This work focuses on the development of an autonomous distributed framework for cooperation amongst a set of MGs without support from a traditional centralized grid. This work proposes a distributed algorithm that supports autonomous operation, enhances cost efficiency, and increases reliability of the overall system. This paper uses a complete information game theoretic approach via coalition formation formulation. The effects of rate policies on the dynamics of cooperation are also investigated in this work. The proposed distributed algorithm empowers the MGs to coordinate and match significant part of energy demand and supply with cooperating MGs. Results of this work show cooperation gains for the microgrid network after implementing the proposed algorithm.
\end{abstract}

\section{INTRODUCTION}

Microgrids (MG) are now a widely acceptable smaller scale power networks that are capable of supplying local controllable loads and utilizing Distributed Energy Resources (DERs) as well as energy storage devices [1]. MGs not only could help alleviate power demand on the grid, but also help save the losses along transmission and distribution line as generation is in more proximity to the load. Microgrids proved to be valuable in their resilience and reliability specially during blackouts and major grid instabilities.

MGs and the newly emerging nanogrids are being positioned to represent modulator building blocks for future smart grid distribution. Many industries are already implementing projects for remote areas where the power delivery solely depends on MGs and DERs [2], [3]. In such areas connectivity to the power grid might be available or not based on proximity to urban infrastructure and degree of investment needed to facilitate the connectivity.

Storage technologies including batteries, EV batteries, flywheels and compressed air banks are being deployed in various parts of the smart grid where they enable solutions such as frequency and voltage regulation, load shifting and shaving. In microgrids, the benefits of utilizing energy storage devices for better utilization of intermittent renewables such as wind have been studied [4]. Zamora and Srivastava [5] reiterate that a microgrid requires energy storage systems to solve the mismatch problems, and to optimally utilize renewable energy while addressing variability and intermittency of these resources.
The power availability and reliability of isolated deployments of MGs, specially with intermittent DERs, could be improved by promoting cooperative or coordinated operation between the different MGs, to arrive at a set of common goals for the collective sets of MGs such as lower losses or lower cost of energy. This is specially appealing for a community of microgrids, as it provides a framework within which cooperation enhances power availability and reliability of the community.

A Microgrid Network (MGN) is composed of a group of microgrids that are capable of interacting with each other on both the physical and cyber levels. From a general perspective an MGN could operate in two modes:

- Coordinated/integrated mode: MGs are connected to the grid to supplement demand and absorb surplus;

- Independent mode (off-grid): in this mode the MGs are totally isolated from the grid, and this traditionally required the MG to internally balance its own energy needs, which would result in reducing load in case of shortage, and reducing (or dumping in case of renewables) excess power generation.

MGNs provide an opportunity for researchers to consider new design schemes that are not possible with the grid. Previous work looked at different interactions between microgrids, Boroyevich et al. [6] propose a hierarchy for interconnecting nanogrids and microgrids and the grid with dynamically electronically decoupled subsystems. In [7] the smart distribution system is clustered into small microgrids, which are scheduled with the main grid by distributed optimizing control. Minciardi and Sacile [8] propose a network of lattice interconnected microgrids of which one is connected to the main grid, and uses centralized optimal control to formulate the scheduling of internal energy storage devices to improve the performance of the overall network. Wu and Guan [9] again consider the sharing of resources and information between microrigds and suggest a decentralized partially-observable Markov decision process for the optimal control of the MGs. The model is based on partial or local information of the system. Shahnia et al. [10] propose coordination between multiple MGs where DERs can supply their own local load and a load in another MG, and they include a physical model with distribution static compensator to help regulate the voltage. Most of the work referenced here proposed sharing internal information of local MG parameters to help facilitate the coordination, in addition 
most of the results were shown for a grid connected MGN, with limited number of interconnected MGs.

In this paper we consider the work presented by Saad et al. [11] and Wei et al. [12] in which authors look at cooperation between microgrids with grid connectivity, for which cooperation is motivated by minimizing energy losses over distribution lines using coalition formation game theory. We provide a system model for the MGN in a grid independent mode, most suitable for remote and urban areas, and we base our model on the assumption that MGs coordinate their cooperation with the minimum information needed for cooperation, hence preserving privacy and enhancing simplicity of the model. We also extend our attention to the dynamics of the cooperation in the model and the interpretation of these dynamics.

The contributions of this work are summarized as follows:

1) Develop a system model for autonomous cooperative MGN with no grid connectivity.

2) Design a distributed algorithm for the cooperative MGN controllers in the aforementioned system model.

This paper is organized as follows. Section II covers the preliminaries of coalition formation games. The problem and proposed system model are described and discussed in III. Simulation results and their discussion are presented in Section IV. Conclusions and final remarks are shown in Section V.

\section{CoAlition Formation BACKGROUnd}

This paper considers cooperation between MGs using a game theoretic based approach. Coalition Formation Games (CFGs) provide a suitable framework to study cooperation in large scale networks, and help answer questions such as which coalitions will emerge, coalition structure characteristics, how do the structure of the formation change in response to variations in externalities.

CFG theory helps a set of $N$ players who want to strengthen their position in a game by forming cooperative coalitions. A coalition game is uniquely defined by $(N, v)$, where $v$ is the coalition value (characteristic function). It is helpful to notice that although players are still self-interested, the focus of CFG is on the group or coalition of players, thus CFGs deal with two notions of rationality: individual rationality (where players seek the best payoff) and collective rationality (where only stable groups will emerge for which the coalition value is the maximum).

Mathematically, for a coalition game with transferable utility (TU) and $N$ finite number of players, the coalition value function $v: 2^{N} \rightarrow \mathbb{R}$ assigns a value for each coalition $S \subseteq N$ with $v(\varnothing)=0$ [13]-[15]. The coalition value function $v$ describes the maximum collective payoff a set of players gain by forming a coalition. For a TU game, the benefit of cooperation is described by Eq. (1) where for any disjoint coalitions $S_{1}$ and $S_{2}$, if $S_{1} \cup S_{2}$ forms, then $S_{1}$ and $S_{2}$ would at least gain the sum of their gains acting individually.

$$
\begin{gathered}
v\left(S_{1} \cup S_{2}\right) \geq \quad v\left(S_{1}\right)+v\left(S_{2}\right), \forall S_{1} \subset N, S_{2} \subset N, \\
\text { s.t. } S_{1} \cap S_{2}=\varnothing
\end{gathered}
$$

We consider the characteristic form of CFGs where the value of cooperation depends on which MGs are members of the formed coalitions. An imputation is a game outcome (possible solution), which is a payoff distribution vector $x=$ $\left\{x_{1}, x_{2}, \ldots, x_{n}\right\}$ among $N$ players, that satisfies:

1) Efficiency, $\sum_{i}^{N} x_{i}=v(N)$, also called collective rationality or group rationality.

2) Individual rationality, $x_{i} \geq v(i)$, for all $i=1, \ldots, N$.

A good payoff vector is one that is stable; where members have no incentive to leave a coalition and seek other coalitions with better payoff for the member, and fair; where the payoff vector is distributed between coalition member in away that relates to the member contribution to the overall coalition value. For Coalition formation games the core solution concept is defined as the set $C$ of stable imputations that satisfies group and individual rationality as defined in Eq. (2).

$$
\begin{aligned}
C= & \left\{x=\left(x_{1}, \ldots, x_{n}\right): \sum_{i \in N} x_{i}=v(N)\right. \\
& \text { and } \left.\sum_{i \in s} x_{i} \geq v(S), \text { for all } S \subset N\right\}
\end{aligned}
$$

To arrive at solutions for a coalition formation game means to find a stable structure that lies in the core, and it is generally a complex problem. An $N$ number of agents have $2^{N}$ possible coalition structures (partitions). Following an exhaustive search approach is of an exponential complexity as a function of the number of agent [16]. One approach to reduce the complexity is to reduce the search space of candidate coalition structures to be evaluated [17], [18]. Algorithmic approaches have been used to arrive at stable structures that lay in the core with a much lower complexity [14], [19], [20]. Apt et al. formalized preference relations between partitions, and described an algorithmic approach that utilizes progressive merge and split rules to transform partitions [14].

The merge-split algorithm in [14] relies on the concept of Pareto order as a comparison relation to formalize preference between partitions. In the following Pareto order definition, a collection refers to a coalition formation structure where coalitions are disjoint.

Definition 1 (Pareto Order [11], [14]). Let the same players $\mathcal{N}=\{1, \ldots, N\}$ be formed in two collections of disjoint coalitions, collection $\mathcal{C}=\left\{C_{1}, \ldots, C_{l}\right\}$ and collection $\mathcal{K}=$ $\left\{K_{1}, \ldots, K_{n}\right\}$ and let the payoff of a player $j$ in a coalition $C_{j} \in(C)$ be defined as $\phi_{j}\left(C_{j}\right)$. Collection $\mathcal{C}$ is preferred over $\mathcal{K}$ by Pareto order, if and only if the payoff of at least one player $j$ under $\mathcal{C}$ is better than the payoff under $\mathcal{K}$ for all players where $j \in\{1, \ldots, N\}$, mathematically:

$$
\mathcal{C} \triangleright \mathcal{K} \Longleftrightarrow\left\{\phi_{j}(\mathcal{C}) \geq \phi_{j}(\mathcal{K}) \forall j \in \mathcal{C}, \mathcal{K}\right\}
$$

Accordingly, the merge-split rules can be described using the Pareto order as follows:

- Merge:

$$
\begin{aligned}
&\left\{T_{1}, \ldots, T_{k}\right\} \cup P \rightarrow\left\{\cup_{j=1}^{k} T_{j}\right\} \cup P, \\
& \text { where }\left\{\cup_{j=1}^{k} T_{j}\right\} \triangleright\left\{T_{1}, \ldots, T_{k}\right\}
\end{aligned}
$$


- Split:

$$
\begin{aligned}
\left\{\cup_{j=1}^{k} T_{j}\right\} \cup P & \rightarrow\left\{T_{1}, \ldots, T_{k}\right\} \cup P, \\
& \text { where }\left\{T_{1}, \ldots, T_{k}\right\} \triangleright\left\{\cup_{j=1}^{k} T_{j}\right\}
\end{aligned}
$$

\section{System MODEL}

Assume a distribution environment with $N$ microgrids, as shown in Fig. 1. Each MG is parameterized by its:

- Power requirements; positive value to imply surplus, and negative value to imply shortage.

- Location; this translates to physical distances between $M G_{i}$ and other components in the system.

- Power rates; the unit price of power exchanged between a consumer and provider MG pair. In this work symmetrical rates $\left(R_{1}\right)$ are assumed for all MGs to simplify the analysis and provide an overall insight.

In the proposed cooperative MGN (CMGN) each MG has an internal controller (MCC) to balance its demand-supply, as well as support external power exchange. The network of interconnected MGs is managed by the distributed CMGN Control Centers (MNCC). All MGs are allowed to exchange power over the shared power network. The model utilizes a global energy storage (GS) for the MGN to facilitate the coordination of energy exchange and to support the balance of the MGN. The global storage has a policy which provides two rates, $R_{2}$ and $R_{3}$ for energy supplied to the storage and energy consumed from the storage respectively.

CMGN can work in two modes; a non-cooperative mode and a cooperative mode. In the non-cooperative MGN, each MG interacts with the GS to supply or consume energy. The existence of the GS provides the means through which the surplus from one MG could provide for the energy needs of another MG. In the cooperative mode MGs self-organize or cluster in a distributed manner into coalitions. Consumer and producer MGs in a coalition coordinate power exchange between members of the coalitions through their MNCCs and if further energy is needed or there is a surplus the resulting coalition interacts with the GS to balance the overall CMGN.

Let a CMGN initialize with $N$ MGs where each $\mathrm{MG}$ is classified, according to its power requirements at time $t$, to be either a consumer or producer MG. Consequently let $S_{\text {cons }}$ represent the subset of consumer MGs and $S_{\text {prod }}$ be the subset of producer MGs. Let $\mathcal{P}$ be defined as the amount of power exchanged during cooperative coalition formation process, and let $P_{i}$ be the power requirements of $M G_{i}$. The cooperative coalition formation game of $N$ MGs, is defined by:

$$
(N, v), v: 2^{N} \rightarrow \mathbb{R}, \forall S \subseteq N, v: \text { total benefit for } S
$$

For every coalition $S$, given an order $\tau \in \Gamma$, where $\Gamma$ is the set of all different orderings of consumers in $S_{\text {cons }} \in S$, the utility of coalition $S$ is expressed in Eq. (3) where the utility is described in terms of overall cost of power for coalition $S$. The overall cost depends on power rates and amounts of power exchanged. The rates $R_{1}, R_{2}$ and $R_{3}$ are the energy rates for power exchange between MGs, power exchange between consumer MGs and the storage, and power exchange between producer MGs and the storage, respectively. Power exchange between consumer MGs and the storage is denoted by $\mathcal{P}_{i s}$, similarly power exchange between producer MGs and storage is denoted as $\mathcal{P}_{j s}$.

$$
\begin{gathered}
u(S, \tau)=\sum_{\substack{i \in S_{\text {cons }} \\
j \in S_{\text {prod }}}} R_{1} \mathcal{P}_{i j}+ \\
\sum_{i \in S_{\text {cons }}} R_{2} \mathcal{P}_{i s}+\sum_{j \in S_{\text {prod }}} R_{3} \mathcal{P}_{j s} \\
v(S)=\min _{\tau \in \Gamma} u(S, \tau)
\end{gathered}
$$

The dynamics of CMGN cooperation is affected by both energy rates and energy losses. The different energy rates can be engineered to control the dynamics of cooperation as shown by results in Section IV. Losses over the distribution lines are calculated according to $P_{i j}^{\text {loss }}=R_{i j} I_{i}^{2}=\frac{P_{i}^{2} R_{i j}}{U_{1}^{2}}$, where $U_{1}$ is the voltage on the distribution line, $R_{i j}$ the resistance of the distribution line between $M G_{i}$ and $M G_{j}$ and is a function of distance between MGs, $I_{i}=\frac{P_{i}}{U_{1}}$ is the current in the distribution line during the power exchange, and $P_{i}$ is the total amount of power exchange. Similar calculations apply for the losses between $M G_{i}$ and the GS.

In this work we adopt the algorithmic approach to arrive at a stable coalition structure. We propose a modified merge-split algorithm that we term Distributed Merge-Swap Algorithm outlined in algorithm 1. In the algorithm description, let $S\left(M G_{i}\right)$, where $\left|S\left(M G_{i}\right)\right| \geq 1$, be defined as the coalition $S$ containing $M G_{i}$ as one of its members. The algorithm initializes with $N$ coalitions being formed, where each coalition contains one MG (which is equivalent to the non-cooperative MGN scenario). The swap step is defined as in (5).

The algorithm loops over the list of consumer MGs, where for each cons $(i), i=\left\{1, \ldots, N_{\text {cons }}\right\}$, the following is done: the producers are ordered according to their corresponding losses if exchanging power with cons $(i)$, with the most preferred producer MG $\operatorname{prod}(m)$ being the one with lowest losses. Check if $\operatorname{prod}(m)$ has enough available power to meet the needs of cons $(i)$; if yes, check the utility of cons $(i)$ if when joining $S(\operatorname{prod}(m))$ has a benefit to $\operatorname{cons}(i)$, then it joins the coalition containing $\operatorname{prod}(m)$. If the most preferred producer does not have enough available power to meet the power needs of cons $(i)$, the algorithm checks whether the maximum power capability of $\operatorname{prod}(m)$ is the available power, if that is the case then $\operatorname{cons}(i)$ joins the coalition containing $\operatorname{prod}(m)$. The same process is repeated for the next preferred $\operatorname{prod}(m)$. If the most preferred producer does not have enough available power to meet the power needs of cons $(i)$ and maximum power capability of $\operatorname{prod}(m)$ is more than the available power, the algorithm retrieves all current consumers in the same coalition with $\operatorname{prod}(m)$, and enumerates to find the consumer with lowest payoff $(\operatorname{cons}(w))$, then it evaluates if a swap could be done based on Pareto order and utility calculations between $S(\operatorname{prod}(m), \operatorname{cons}(w))$ and $S(\operatorname{prod}(m), \operatorname{cons}(i))$. Then, the algorithm iterates until the number of swaps converges to zero 


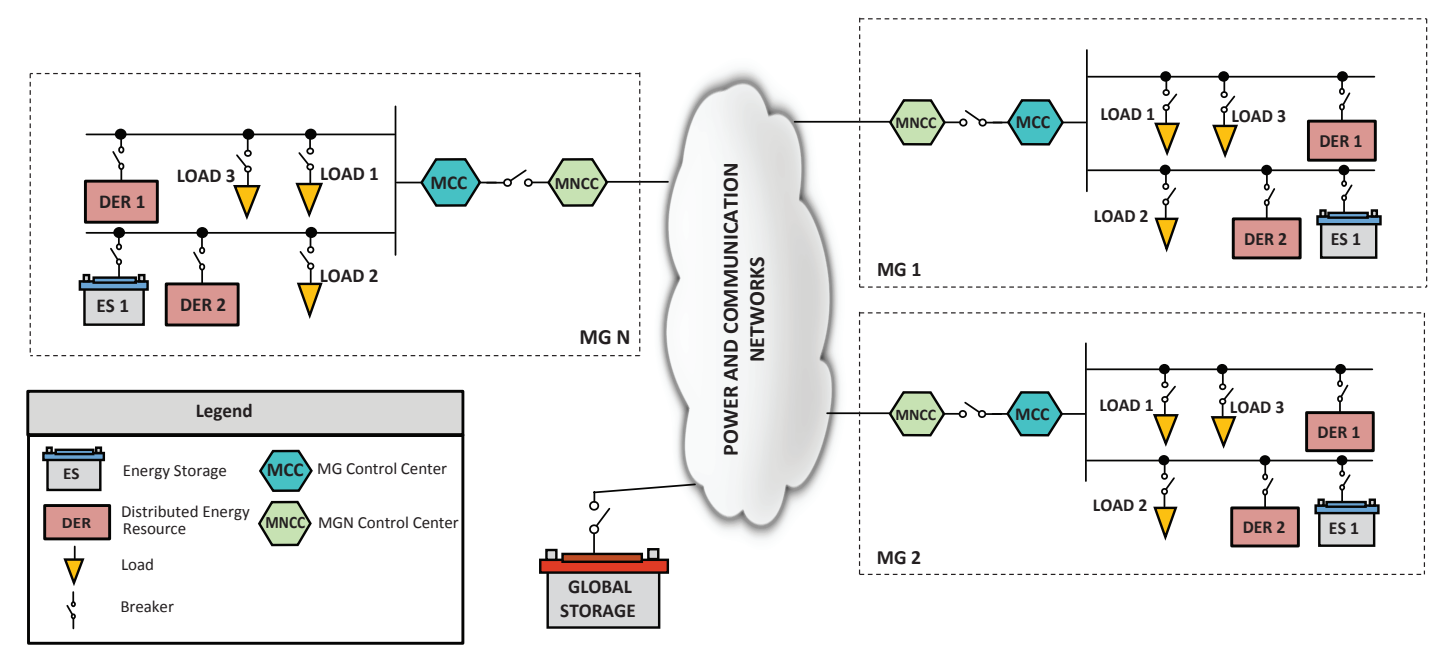

Fig. 1. Cooperative Microgrid Network

indicating a stable coalition structure. The swap step is defined as in Eq. (5).

$$
\begin{aligned}
& \text { Swap }(\operatorname{cons}(i), \operatorname{prod}(m), \operatorname{cons}(w))= \\
& \left\{\begin{array}{l}
S(\operatorname{prod}(m), \operatorname{cons}(w)) \rightarrow S(\operatorname{prod}(m)),\{\operatorname{cons}(w)\} \\
\& \\
S(\operatorname{prod}(m)) \cup \operatorname{cons}(i) \rightarrow S(\operatorname{prod}(m), \operatorname{cons}(i))
\end{array}\right.
\end{aligned}
$$

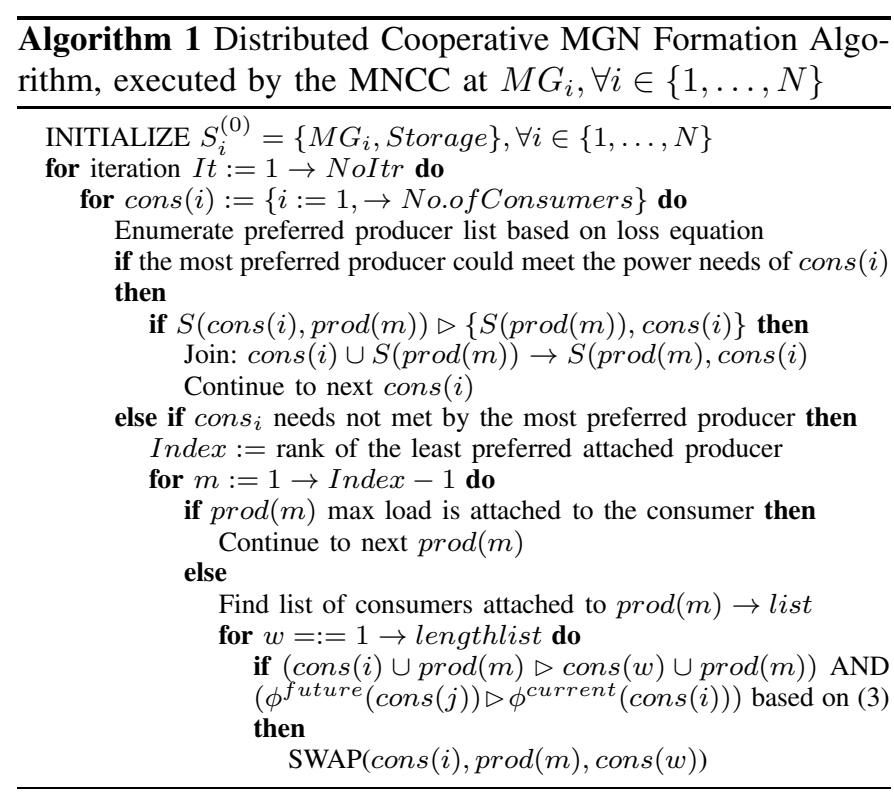

The analysis provided above can easily extend to the operation of the cooperative MGN in a dynamic setting, where changes in the dynamics of the network (for example varying power requirements of MGs) will trigger the algorithm to recoordinate the cooperation. Proposed CMGNs are also beneficial in enhancing the resilience of the overall MGN against loss of producer or consumer MGs, as the algorithm will treat the loss as a change in the dynamics and consequently affected MGs will seek a different coalition formation structure.

For our CMGN, we lay here the assumptions underlying the system model; all MGs have full communication and power line connectivity. Each MG is assumed to have an internal controller that is capable of regulating and maintaining frequency and voltage stability while supporting power trading with external MGs, but an MG cannot be a consumer and a producer at the same time. All MGs have access to the global storage, and the storage is assumed to be able to support multiple energy transactions at the same time.

\section{A. Convergence and stability-sketch proof}

Algorithm 1 builds on the merge and split algorithm [14] where the swap step is based on the comparison preference of Pareto order; based on the proof provided in [14], it is guaranteed to converge to a stable structure if one exists.

\section{B. Cooperation Measures}

To evaluate the benefits of cooperation for CMGN, it is insightful to define cooperation gain as a measure of the cooperation benefit. We define cooperation gain as the normalized difference between non-cooperative power cost and cooperative cost, as in Eq. (6) where the non-cooperative power cost is calculated based on $N$ individual MGs that interact directly with the storage as shown in Eq. (7), and the cooperative power cost is based on the value of the cooperative coalition formation game, defined by Eq. (3) and Eq. (4).

$$
\text { Cooperation Gain }=\frac{\text { Non-cooperative cost }- \text { Cooperative cost }}{\text { Non-cooperative cost }}
$$

Non-cooperative Cost $=\sum_{i \in S_{\text {cons }}} R_{2} P_{i s}+\sum_{j \in S_{\text {prod }}} R_{3} P_{j s}$

As we include the energy storage to facilitate the interaction between the individual MGs in the MGN, it is valuable 
to study how dependent would the distributed cooperation be on the storage. With interesting insights as how could this help the system designers determine the suitable storage capacity needed to support the cooperative MGN. Let the Storage Dependency Ratio (SDR) be defined by Eq. (8) for the cooperative MGN, it is clear from this definition that the non-cooperative SDR is equal to 1 .

$$
\mathrm{SDR}=\frac{\max \left\{\sum_{i} \mathcal{P}_{i s}, \sum_{j} \mathcal{P}_{j s}\right\}}{\max \left\{\sum_{i} P_{i s}, \sum_{j} P_{j s}\right\}}
$$

\section{Numerical Results AND Discussion}

In this section different scenarios are simulated to provide further insight into the dynamics of cooperation and the algorithm performance. All quantities with no loss of generality are in per unit bases. $N$ MGs are randomly divided into two subsets of consumers and producers with corresponding random energy requirements of a value between 0 and 100 . MGs are randomly placed on a grid of 10x10, with the GS placed at the center. Power losses in power lines between MGs and between MGs and GS are calculated based on a line resistance of $R=0.1$ and line voltage $U_{1}=1$. To obtain representative results, the process is repeated for a large number of iterations, and the various measures are averaged over the number of iterations. GS capacity is assumed to be unlimited to facilitate the study of the dynamics of the system which would help provide an insight into the capacity design of the GS.

\section{A. Performance vs. Number of MGs}

To study the performance of the algorithm the number of MGs is varied between 12 and 202 and power rates are fixed at $R_{1}=1, R_{2}=2, R_{3}=1$, then various metrics are calculated as shown in Fig. 2 and Fig. 3. Interestingly, cooperation gain shows to be robust to the number of MGs in the network at a value around $50 \%$ for the fixed power rate policy given, and this result hints that the algorithm is always able to arrive at an optimal structure that results in a substantial cooperation gain over the non-cooperative case for a given rate policy. SDR results show a similar behaviour and confirm that a GS of capacity equal to the net difference between overall supply and demand of the MGN is enough to support the cooperative MGN under normal operating conditions.

Algorithm performance results in Fig. 3 show that the algorithm run time increase vs. $N$ follows a polynomial function of second order which is an advantage for the algorithmic approach versus exhaustive methods. We can also observe from Fig. 3 that the maximum size of a coalition is also robust to $N$ given a fixed rate policy. The above results emphasize the role of the power rate policy in the dynamics of the cooperation, we further explore this in the subsequent set of simulations.

\section{B. Effect of Rate Policy}

To study the effect of power rate policy on the dynamics of the cooperative MGN, the second set of simulations is run for two power rate policies. In the first policy $R_{3}=2$ is fixed
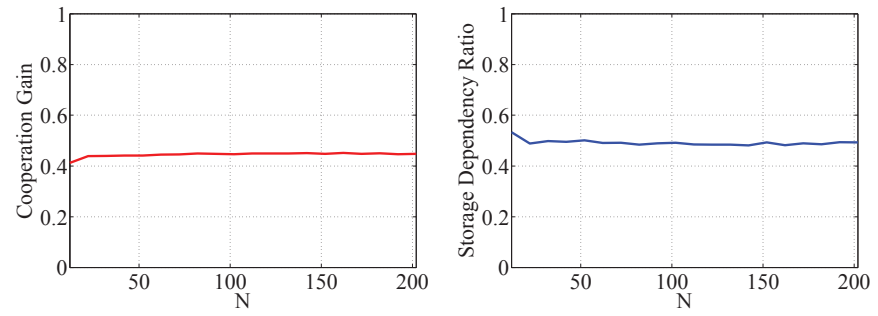

Fig. 2. Cooperative gain and SDR vs. $\mathrm{N}$ for fixed $R_{1}=1, R_{2}=2, R_{3}=1$
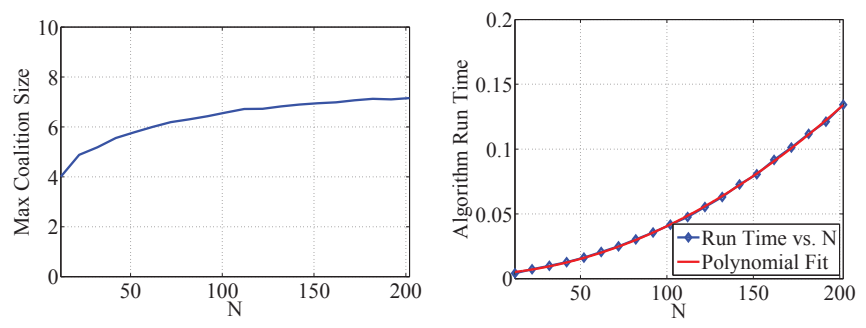

Fig. 3. Maximum coalition size and algorithm run time vs. $\mathrm{N}$ for fixed $R_{1}=1, R_{2}=2, R_{3}=1$

as well as $N=20$ while $R_{1}, R_{2}$ are varied between 0 and 4, and results are shown in Fig. 4. It is clear that cooperation is strongly discouraged for high values of $R_{1}$ in the region where $R_{2}$ is lower than $R_{1}$ and $R_{3}$ which is expected as the relatively lower storage rates compared to local exchange rates would result in MGs tending to depend on the storage GS. Cooperation dynamics are quite interesting for values of $R_{1}$ less than $R_{3}$ where cooperation gain and SDR clearly improve as a result.

Further we study another rate policy in which $R_{1}=1$ is fixed and GS storage rates $R_{2}, R_{3}$ are varied between 0 and 4 . The results shown in Fig. 5 for cooperation gain and number of coalitions confirm that $R_{1}$ has a higher impact on the dynamics of the coalition formation and the cooperation in the MGN. Cooperation gain and average number of coalitions were almost constant in the region $R_{2}, R_{3}>R_{1}=1$ which means that the storage policy either discourages or encourages the cooperation, but $R_{1}$ directly impacts the dynamics of the coalition formation process and effectiveness, and this suggests that MGs could effectively design their power rates to harness the best benefit.

\section{CONCLusions}

This work proposes a cooperative microgrid network for remote and rural areas that is independent of the main grid, and formulates a distributed algorithm for cooperation that is based on coalition formation games. We study the ability of global storage to facilitate cooperation amongst the MGs within the network. The objective of proposed system is to improve individual MG demand-supply balance by cooperation, as well as to best utilize renewable DER by exchanging surplus with cooperating MGs and provide resiliency to loss of member 


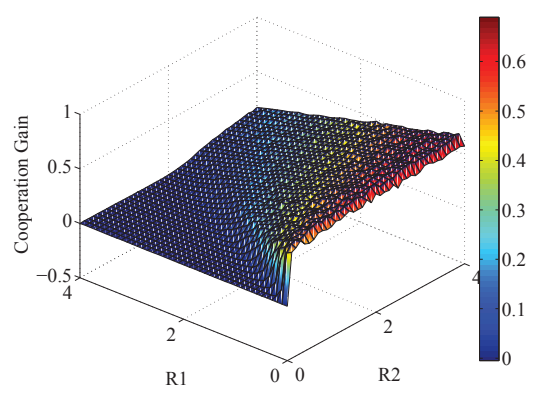

(a) Cooperation gain

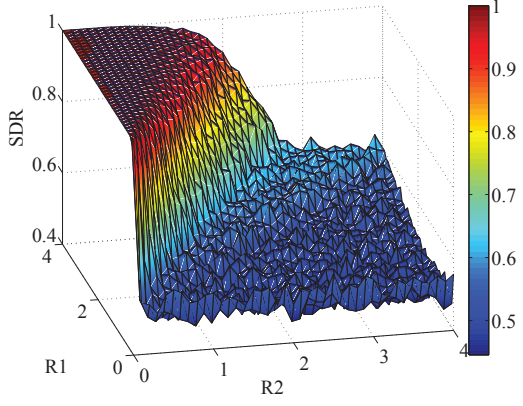

(b) Cooperation SDR

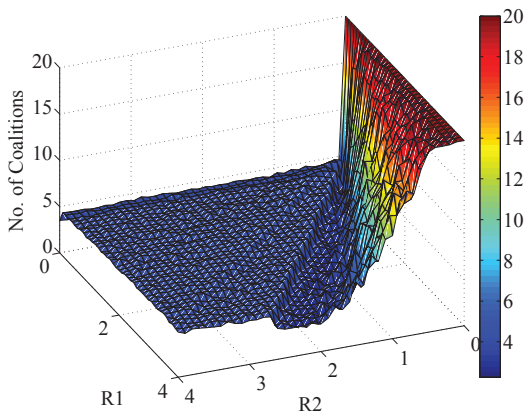

(c) Average number of coalitions

Fig. 4. Cooperation gain, SDR ratio and average number of coalitions for fixed $R_{3}=2, N=20$ and variable $R_{1}, R_{2}$.
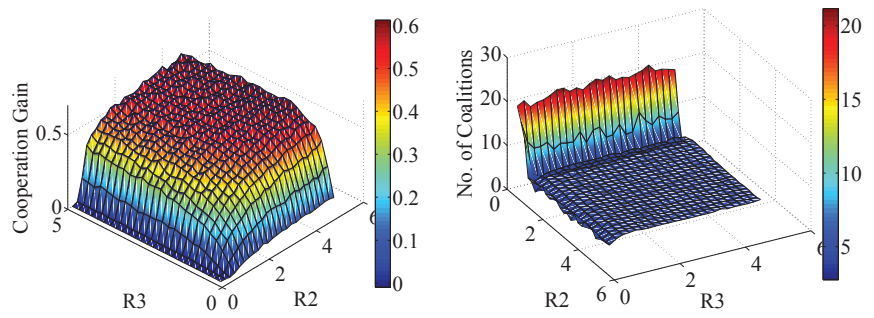

Fig. 5. Cooperation gain and number of coalitions for fixed $R_{1}=1, N=22$ and variable $R_{2}, R_{3}$

MGs. This paper also defined measures for cooperation including cooperation gain and storage dependency ratio.

Numerical results demonstrate the benefits of employing this cooperative model, and provide insights into the dynamics of cooperation. The rate policy role in the dynamics of cooperation has been studied as well. Results were also shown for algorithm performance and robustness.

\section{REFERENCES}

[1] N. Hatziargyriou, H. Asano, R. Iravani, and C. Marnay, "Microgrids," IEEE Power and Energy Magazine, vol. 5, no. 4, pp. 78-94, 2007.

[2] G. Kyriakarakos, A. I. Dounis, S. Rozakis, K. G. Arvanitis, and G. Papadakis, "Polygeneration Microgrids: a viable solution in remote areas for supplying power, potable water and hydrogen as transportation fuel," Applied Energy, vol. 88, no. 12, pp. 4517-4526, 2011.

[3] D.-R. Thiam, "Renewable decentralized in developing countries: Appraisal from microgrids project in Senegal," Renewable Energy, vol. 35, no. 8 , pp. 1615-1623, 2010.

[4] J. P. Barton and D. G. Infield, "Energy Storage and Its Use With Intermittent Renewable Energy," IEEE Transactions on Energy Conversion, vol. 19, no. 2, pp. 441-448, 2004.

[5] R. Zamora and A. K. Srivastava, "Controls for Microgrids With Storage: Review, Challenges, and Research Needs," Renewable and Sustainable Energy Reviews, vol. 14, no. 7, pp. 2009 - 2018, 2010.

[6] D. Boroyevich, I. Cvetkovic, R. Burgos, and D. Dong, "Intergrid: A Future Electronic Energy Network?" 2013.

[7] R. H. Lasseter, "Smart Distribution: Coupled Microgrids," Proceedings of the IEEE, vol. 99, no. 6, pp. 1074-1082, 2011.

[8] R. Minciardi and R. Sacile, "Optimal Control in a Cooperative Network of Smart Power Grids," Systems Journal, IEEE, vol. 6, no. 1, pp. 126133, 2012.

[9] J. Wu and X. Guan, "Coordinated Multi-Microgrids Optimal Control Algorithm for Smart Distribution Management System," Smart Grid, IEEE Transactions on, vol. 4, no. 4, pp. 2174-2181, Dec 2013.
[10] F. Shahnia, R. P. Chandrasena, S. Rajakaruna, and A. Ghosh, "Autonomous Operation of Multiple Interconnected Microgrids with SelfHealing Capability," in 2013 IEEE Power and Energy Society General Meeting (PES). IEEE, 2013, pp. 1-5.

[11] W. Saad, Z. Han, and H. V. Poor, "Coalitional Game Theory for Cooperative Micro-Grid Distribution Networks," in 2011 IEEE International Conference on Communications Workshops (ICC). IEEE, 2011, pp. $1-5$.

[12] C. Wei, Z. Fadlullah, N. Kato, and A. Takeuchi, "GT-CFS: A Game Theoretic Coalition Formulation Strategy for Reducing Power Loss in Micro Grids," IEEE Transactions on Parallel and Distributed Systems, vol. PP, no. 99, 2013.

[13] R. P. Gilles, The Cooperative Game Theory of Networks and Hierarchies. Springer, 2010, vol. 44.

[14] K. R. Apt and A. Witzel, "A Generic Approach to Coalition Formation," International Game Theory Review, vol. 11, no. 03, pp. 347-367, 2009.

[15] W. Saad, Z. Han, M. Debbah, and A. Hjrungnes, "Coalitional game theory for communication networks: A tutorial," in IEEE Signal Processing Magazine.

[16] T. Rahwan, S. D. Ramchurn, N. R. Jennings, and A. Giovannucci, "An Anytime Algorithm For Optimal Coalition Structure Generation," Journal of Artificial Intelligence Research, vol. 34, no. 2, p. 521, 2009.

[17] T. Rahwan, "Algorithms for Coalition Formation in Multi-Agent Systems," Ph.D. dissertation, University of Southampton, 2007.

[18] O. M. Shehory, K. Sycara, and S. Jha, "Multi-Agent Coordination Through Coalition Formation," in Intelligent Agents IV Agent Theories, Architectures, and Languages. Springer, 1998, pp. 143-154.

[19] P. Caillou, S. Aknine, and S. Pinson, "A Multi-Agent Method for Forming and Dynamic Restructuring of Pareto Optimal Coalitions," in Proceedings of the first international joint conference on Autonomous agents and multiagent systems: part 3. ACM, 2002, pp. 1074-1081.

[20] K. R. Apt and T. Radzik, "Stable Partitions in Coalitional Games," arXiv preprint cs/0605132, 2006. 